\title{
On Addition Schemes
}

\author{
Srećko Brlek ${ }^{1}$ \\ LACIM, Dépt. de Math. et Info., Univ. du Québec à Montréal, \\ C.P. 8888, Succ. A, Montréal H3C 3P8 Canada. \\ Pierre Castéran ${ }^{2} \quad$ Robert Strandh $^{3}$ \\ LABRI, U.F.R Math. et Info., Université Bordeaux I, \\ 33405 Talence CEDEX, France.
}

\begin{abstract}
We review a class of addition chains that are suboptimal but close to optimal. Addition chains in this class are generated by a very efficient algorithm. We present evidence that traditional algorithms for exponentiation may in fact be slower than a method where an addition chain is first constructed, then applied and finally discarded. Experimental evidence definitely indicates this to be true for slightly more complex domains than the integers.

To achieve such a result, we define an interpretation of addition chains as sequences of function applications using continuation passing style which can be compiled by transformation into sequences of simple "combinators". Finally, we propose optimization techniques for addition chains in this representation.
\end{abstract}

keywords: addition chains, continuations, programming languages semantics, compilation of functional languages, Scheme programming language, semi-numerical algorithms.

\section{INTRODUCTION}

The problem of computing $x^{n}$ for a given integer $n$ in some monoid is crucial in a number of algorithms for numeric as well as symbolic calculations. If $n$ is a constant known at compile time, or if we know that $x^{n}$ will be computed several times for different values of $x$ but with the same $n$, a good method is to generate a computation scheme with a minimal number of multiplications. This method is also worthwhile in the case of complex multiplications (bignums, polynomials, matrices,...). The concept of addition chain has been introduced in [15] to deal with the complexity of the multiplication scheme:

- An addition chain for a positive integer $n$ is a sequence $\sigma=\left\langle n_{1}, n_{2}, \ldots, n_{m}\right\rangle$ of positive integers such that:

$-n_{1}=1, n_{m}=n$,

$-\forall i, 1<i \leq m, \exists j, k$ such that $n_{i}=n_{j}+n_{k}$.

1 With partial support from NSERC-Canada and FCAR-Québec, e-mail: brlek@lacim.uqam.ca

2 With the support of PRO "GRECO de Programmation", e-mail: casteran Qgeocub.greco-prog.fr

3 With the support of PRC "GRECO de Programmation", email: strandh@geocub.greco-prog,fr 
- The length of the chain $\sigma$ is the number $m$. The chain length $\ell(n)$ of $n$ is the minimal length of all possible chains for $n$.

Thus, addition chains are immediately related to the problem of computing $x^{n}$, since $n_{i}=n_{j}+n_{k}$ implies $x^{n_{i}}=x^{n_{j}} \times x^{n_{k}}$.

Clearly the length of an addition chain is equal to the number of multiplications one needs to compute $x^{n}$. Similarly, the smallest number of such multiplications is given by the chain length $\ell(n)$ of $n$.

The subject has a long history, a detailed account of which is given by Knuth in his second volume [13]. Any explicit algorithm for the generation of addition chains cl early sets an upper bound on the function $\ell(n)$. Thus the usual binary expansion algorithm (see Knuth[13] for instance) implies :

$$
\ell(n) \leq \lambda(n)+\nu(n)-1
$$

where $\lambda(n)=\left\lfloor\log _{2}(n)\right\rfloor$ and $\nu(n)$ is the number of " 1 " in the binary expansion of $n$. However, the problem of computing the exact value of $\ell(n)$ seems to be difficult. Indeed, a slightly more complex problem, namely the problem of computing the chain length for a set of integers, has been shown to be $N P$-complete [10]. Therefore, it is interesting to consider sub-optimal addition chains, provided that they can be constructed in an efficient way.

Such a class of sub-optimal addition chains for positive integers $n$ have been introduced $[1,2,3]$. Chains in this class are obtained through continued fraction expansions for $n / k$, where $k$ is some integer chosen between 2 and $n-1$. Chains of this form are called continued-fraction addition chains, or cf-chains, or simply euclidean chains. It was proved in the same papers $[1,2,3]$, that the Scholz-Brauer conjecture holds for cfchains. This result implies that for an infinite class of integers, cf-chains are much closer to optimal addition chains than the chains obtained by the usual binary method. Even though minimal-length euclidean chains are not optimal, they have the nice property of being easy to compute. Moreover, most of the popular effective strategies for computing addition chains are obtained as special cases of the continued fraction method. Thus, minimal length euclidean chains are systematically shorter than the chains obtained by these other methods.

Traditionally, research on addition chains use the standard representation as sequences of integers; this last representation is well fitted for reasoning about chain lengths, a good measure of the efficiency of the various classes of chains.

Nevertheless, we are interested in the efficiency of the entire process of calculating $x^{n}$, i.e. calculating the chain for $n$ as well as applying the result to $x$.

Research on functional/applicative languages, their semantics and implementation, gives us a good formalism for that purpose. In particular, we use the so called "Continuation Passing Style" (CPS) [14,18] which becomes more and more popular among the Scheme-addicts.

Since we reserved the term "addition chain" for the sequences of integers defined above, we use the term "addition scheme" to denote the entire computation process for $x^{n}$ described in this paper. 


\section{Addition Schemes}

\subsection{Star Chains}

Euclidean chains form a sub-class of star chains, i.e, chains verifying the properties:

- $\forall i, n_{i}<n_{i+1} \quad$ (increasing chains)

- $\forall i>1, \exists j<i$ such that $n_{i}=n_{i-1}+n_{j}$.

Star chains can be viewed as iterative algorithms for computing $x^{n}$ the obvious way. Let us take two examples for $n=87$ :

$$
\begin{gathered}
\sigma_{1}=\langle 1,2,3,6,7,10,20,40,80,87\rangle, \\
\sigma_{2}=\langle 1,2,4,5,10,20,21,42,43,86,87\rangle .
\end{gathered}
$$

Figures 1 and 2 show the graphical representations for $\sigma_{1}$ and $\sigma_{2}$ as labeled dags: an arc (left-to-right) between $j$ and $i$ means that $x^{j}$ is used in the computation of $x^{i}$; arcs originated from the same vertex are merged.

The chain $\sigma_{1}$ will be used as the standard example troughout this paper.

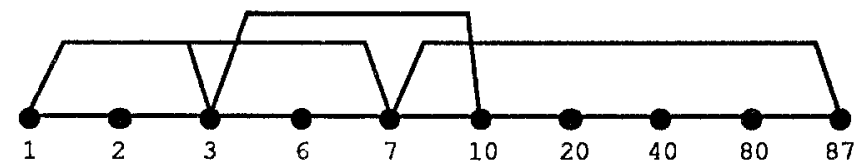

Fig. 1 Dag associated with the chain $\sigma_{1}$

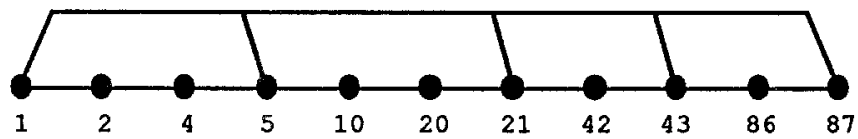

Fig. 2 Dag associated with the chain $\sigma_{2}$

In the rest of this paper, only star chains will be considered. So the term "chain" will denote "a star addition chain".

\subsection{Width of a chain}

The width $w(\sigma)$ of a chain $\sigma$ is the width of the associated dag, it clearly defines the number of registers used to compute $x^{n}$ : for instance, 2 for $\sigma_{2}, 3$ for $\sigma_{1}$. As an example, we show in figure 3 a low-level program to compute $x^{87}$ following $\sigma_{1}$. 


\subsection{Representation Problems}

The representation of a chain as a sequence of integers does not reflect the structure of the computation (alternation of products and squares, width of the chain) and so cannot simply be translated into an efficient low-level program.

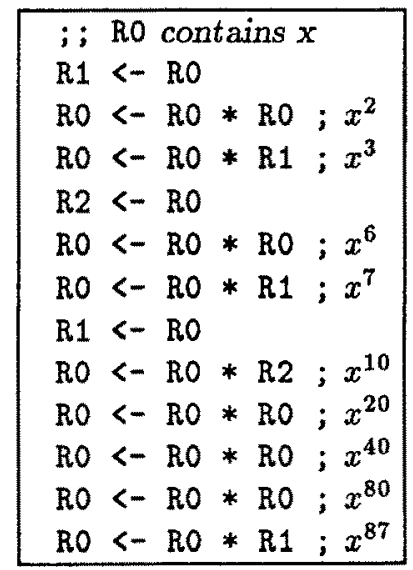

Fig. 3 Low-level program following $\sigma_{1}$

As an example, let us consider the (short) chain $\sigma^{\prime \prime}=\langle 1,2,3,6,7\rangle$, the dag representation of which is given in figure 4 .

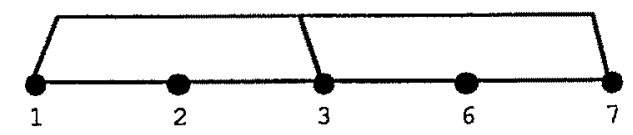

Fig. 4 A representation for $\sigma^{\prime \prime}=\langle 1,2,3,6,7\rangle$

We note immediately that $\sigma^{\prime \prime}$ is not a substructure of $\sigma_{1}$ although one might think so from their respective representation as integer sequences. Effectively the low-level programm associated with $\sigma^{\prime \prime}$ cannot be a part of the one in figure 3. Consequently, we have to consider the structure of the computation defined by a star chain, and not its integer sequence representation, which represents a loss of information. Of course, it is always possible to decode such a sequence into a dag, but this operation can slow the computation, particularly when only one computation of $x^{n}$ is needed. Our representation of star chains, borrowed from denotational semantics and functional languages compilation can give us some advantages:

- The correctness of our algorithms is immediate.

- A lot of research is done on optimization of such languages, and this research can be applied to our goals. 


\subsection{Operational definitions}

In this section, we try to give an intuitive view of cf-chains, by a description of how $x^{n}$ is computed, following suh a chain. The precise (functional) definition will be given in 3.3.

\subsubsection{Strategies}

The definition of a computation scheme for $n$ is parametrized by a component called strategy, i.e a function $\gamma$ which determines for each positive integer $n$ (which is not a power of 2) some non-empty subset $\gamma(n)$ of $\{2,3, \ldots, n-1\}$. Among all possible strategies, we can cite:

- The binary strategy is defined by:

$$
\beta(n)=\left\{\left\lfloor\frac{n}{2}\right\rfloor\right\} .
$$

$\beta$ defines exactly the binary method for computing $x^{n}$, in Knuth's terminology.

- The total strategy $\theta$ corresponds to the choice of all possible candidates, i.e.:

$$
\theta(n)=\{2,3, \ldots, n-1\} .
$$

- The dichotomic strategy $\delta$ is defined by:

$$
\delta(n)=\left\{n \operatorname{div} 2^{k}, \text { with } k=\lceil\lambda(n) / 2\rceil\right\} .
$$

A strategy $\gamma$ is said to be deterministic if $\gamma(n)$ is a singleton for each $n$. The binary and dichotomic strategies are deterministic. Other strategies are defined and compared in $[1,2,3]$. In these papers, it appears that the dichotomic strategy is the best compromise between the efficiency of chain generation and generated chains. Given a strategy $\gamma$, we will use the locution "a $\gamma$-chain" for "a chain generated with $\gamma$ ".

\subsubsection{Operational Definitions}

The following definitions of computation schemes for $x^{n}$ are parametrized by a strategy $\gamma$. In order to shorten the notations, we assume in the rest of this section that a strategy $\gamma$ is fixed. We need to introduce three kinds of computation schemes:

- $M(x, n):$ computation of $x^{n}$

- $C(x, n, p)$ : computation of $x^{n}$, a step of which is the computation of $x^{p}$

- $K(x, n, p)$ : computation of $x^{n}$ and $x^{p}$.

The main difference between the two last kind of schemes is that in the last one the value of $x^{p}$ is needed by the rest of the computation, and in the other case the computation of $x^{p}$ is just a step of the computation. The relation between the three constructs is given by:

- $M(x, n)$ :

- If $n=3$ then return $x^{2} \times x$.

- If $n=2^{a}$ then compute $x^{2}, x^{4}, \ldots, y=x^{n}$ ( $a$ squares), then return $y$. 
- Otherwise, return $C(x, n, p)$ where $p \in \gamma(n)$

- $C(x, n, p)$ :

- If $n=p u$ then compute $y=M(x, p)$, then return $M(y, u)$.

- If $n=p u+j(0<j<p)$, then compute $(y, z)=K(x, p, j)$, then return $M(y, u) \times z$.

- $K(x, n, p)$ :

- If $n=p u$, then compute $y=M(x, p)$, then return $M(y, u)$ and $y$.

- If $n=p u+j(0<j<p)$, then compute $(y, z)=K(x, p, j)$, then return $M(y, u) \times z$ and $y$.

The definitions above contain some imperative constructs: return, then, and, which are formalized and implemented in the next section.

Remark also the nondeterminism of these definitions; in the case of a nondeterministic strategy the $p$ which minimizes the length of the computations is selected. In this case, $M(x, n)$ defines the computation associated with the minimal chain according to $\gamma$ (cf. the minchain algorithms of $[1,2,7]$.)

\section{Chatns AND Continuations}

Continuations, introduced as a tool for the formalization of control structures [16], are now considered as a practical tool: for instance, first class continuations in Scheme $[11,17]$, compilation and optimization by program transformations $[12,14,18]$. From this research has emerged an interesting programming style, the so called "Continuation Passing Style" (CPS), which consists in giving to some functions an additional parameter representing the rest of the computation.

As an example, consider again the chain $\sigma_{1}$ and analyze the computation of $x^{87}$ for some $x$. Let us arbitrarily break that computation in two parts: one corresponding to the sub-chain $\sigma^{\prime}=\langle 1,2,3,6,7\rangle$ and the other to the chain suffix $\tau=\langle 10,20,40,80,87\rangle$. boxes).

In figure 5 , we show the communications between $\sigma^{\prime}$ and $\tau$ (considered as black

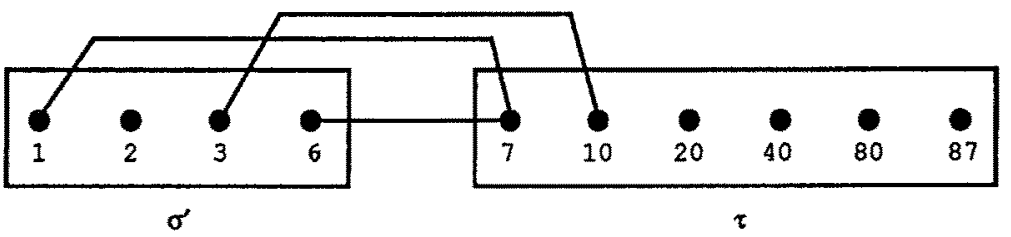

Fig. 5 Communications between two parts of $\sigma_{1}$

We can say that $\sigma^{\prime}$ sends the values $\left(x^{6}, x, x^{3}\right)$ to $\tau$, which receives them, in order to produce the result $x^{87}$. In other words, we consider [sub-]chains as producers of sequences of values, and chain suffixes as receivers of such sequences. In Semantics terminology, we associate with $\tau$ a continuation of the computation associated with $\sigma^{\prime}$. 


\subsection{Notation}

\subsubsection{Values}

D denotes some domain on which a product $\times$ is defined. The metavariables $x, y, z, \ldots$, are used for any element of $\mathbf{D}$.

$\mathbf{D}^{*}$ stands for the domain of sequences $\left\langle x_{1}, \ldots, x_{n}\right\rangle$ of elements of $\mathbf{D}$ (metavariables $\left.: x^{*}, y^{*}, \ldots\right)$.

$\mathbf{D}^{+}$stands for the domain of nonempty sequences of elements of $\mathbf{D}$ (metavariables $\left.: x^{+}, y^{+}, \ldots\right)$.

Sequence appending is denoted by $\S,\left\langle x_{1}, \ldots, x_{n} \cdot x^{*}\right\rangle$ is an abbreviation for $\left\langle x_{1}, \ldots, x_{n}\right\rangle \S x^{*}$

\subsubsection{Functions}

We freely use sequence patterns in lambda-expressions; for instance the equivalent of lisp's cadr function is denoted by $\lambda\left\langle x_{1}, x_{2} \cdot x^{*}\right\rangle \cdot x_{2}$. We often omit parentheses in function applications, and freely use subscripts for some arguments of function applications: for instance $f g h_{n p}\left\langle x \cdot x^{*}\right\rangle$ stands for $\left.(((f g) h) n) p\right)\left(\left\langle x \cdot x^{*}\right\rangle\right)$. The composition of two functions $f$ and $g$ is defined as usual by

$$
(g \circ f)(x)=g(f(x))
$$

\subsection{Main domains}

\subsubsection{Chain continuations}

We define the domain Konts $=\mathbf{D}^{+} \rightarrow \mathbf{D}$ of chain-continuations. Elements of Konts are functional representations of chain suffixes like $\tau$. For instance, we can associate with $\tau$ the function $K_{\tau}: \mathbf{D}^{+} \rightarrow \mathbf{D}$ such that

$$
K_{\tau}\langle x, y, z\rangle=\text { let } t=x \times y \text { in }(t \times z)^{8} \times t .
$$

$K_{\tau}$ is such that $K_{\tau}\left\langle x^{6}, x, x^{3}\right\rangle=x^{87}$.

\subsection{2 (Functional) chains}

Following the definitions in the previous section, it is natural to associate with each chain $\sigma$ a function $F_{\sigma}$ : Konts $\rightarrow$ Konts. To do so, we define the domain Chains $=$ Konts $\rightarrow$ Konts.

For instance we associate with $\sigma_{1}$ and $\sigma^{\prime}$ respectively the two elements of Chains defined by

$$
F_{\sigma_{1}} \kappa\left\langle x \cdot x^{*}\right\rangle=\kappa\left(\left\langle x^{87} \cdot x^{*}\right\rangle\right),
$$

and

$$
F_{\sigma^{\prime}} \kappa\left\langle x \cdot x^{*}\right\rangle=\kappa\left(\left\langle x^{6}, x, x^{3} \cdot x^{*}\right\rangle\right) .
$$

The relation with powering is given by

$$
x^{87}=F_{\sigma_{1}}\left(\lambda\left\langle x \cdot x^{*}\right\rangle \cdot x\right)\langle x\rangle .
$$




\subsection{Formal definitions}

\subsubsection{Functionalities}

We want to define functional objects, associated with the intuitively defined computation schemes $M, C$ and $K$. Their functionality is defined below:

$$
\begin{aligned}
\mathcal{M} & : N_{1} \rightarrow \text { Chains } \\
\mathcal{C} & : N_{1} \rightarrow N_{1} \rightarrow \text { Chains } \\
\mathcal{K} & : N_{1} \rightarrow N_{1} \rightarrow \text { Chains }
\end{aligned}
$$

The intentional definition of these three functions is:

$$
\begin{aligned}
\mathcal{M} & =\lambda n \kappa\left\langle x \cdot x^{*}\right\rangle \cdot \kappa\left\langle x^{n} \cdot x^{*}\right\rangle \\
\mathcal{C} & =\lambda n p \kappa\left\langle x \cdot x^{*}\right\rangle \cdot \kappa\left\langle x^{n} \cdot x^{*}\right\rangle \\
\mathcal{K} & =\lambda n p \kappa\left\langle x \cdot x^{*}\right\rangle \cdot \kappa\left\langle x^{n}, x^{p} \cdot x^{*}\right\rangle .
\end{aligned}
$$

Now we give recursive definition of these three functions. The definition of some auxiliary functions will simplify our work.

\subsubsection{Auxiliary functions}

We define four functions: $\mathbf{W}, \mathbf{W}_{\mathbf{2}}, \mathbf{X}$ and $\mathbf{Q}$ : Chains and a function $\mathbf{K}$ : Konts. The names of the first two functions is inspired by Combinatory Logic (see [8,9]). We will see that their interest results from the fact that they can be implemented very efficiently in terms of elementary stack operations.

$$
\begin{aligned}
& \mathbf{W}=\lambda \kappa\left\langle x_{0} \cdot x^{*}\right\rangle \cdot \kappa\left\langle x_{0}, x_{0} \cdot x^{*}\right\rangle \\
& \mathbf{W}_{\mathbf{2}}=\lambda \kappa\left\langle x_{0}, x_{1} \cdot x^{*}\right\rangle \cdot \kappa\left\langle x_{0}, x_{1}, x_{0} \cdot x^{*}\right\rangle \quad \text { duplication and transpose } \\
& \mathbf{X}=\lambda \kappa\left\langle x_{0}, x_{1} \cdot x^{*}\right\rangle \cdot \kappa\left\langle x_{0} \times x_{1} \cdot x^{*}\right\rangle \quad \text { product } \\
& \mathbf{Q}=\lambda \kappa\left\langle x_{0} \cdot x^{*}\right\rangle \cdot \kappa\left\langle x_{0}^{2} \cdot x^{*}\right\rangle \quad \text { square } \\
& \mathbf{K}=\lambda\left\langle x \cdot x^{*}\right\rangle \cdot x \\
& \text { projection }
\end{aligned}
$$

Now we can give a recursive definition of $\mathcal{M}, \mathcal{C}$, and $\mathcal{K}$ in terms of our auxiliary definitions.

- $\mathcal{M}_{3}=\mathbf{W} \circ \mathbf{Q} \circ \mathbf{X}$

- $\mathcal{M}_{n}=\overbrace{\mathbf{Q} \circ \mathbf{Q} \circ \ldots \circ \mathbf{Q}}^{a \text { times }}$ if $n=2^{a}$

- $\mathcal{M}_{n}=\mathcal{C}_{n p}$ where $p$ is some integer in $\gamma(n)$

- $\mathcal{C}_{n p}=\mathcal{M}_{p} \circ \mathcal{M}_{u}$, if $n=p u$

- $\mathcal{C}_{n p}=\mathcal{K}_{p j} \circ \mathcal{M}_{u} \circ \mathrm{X}$, if $n=p u+j(0<j<p)$

- $\mathcal{K}_{n p}=\mathcal{M}_{p} \circ \mathrm{W} \circ \mathcal{M}_{u}$, if $n=p u$

- $\mathcal{K}_{n p}=\mathcal{K}_{p j} \circ \mathbf{W}_{\mathbf{2}} \circ \mathcal{M}_{u} \circ \mathbf{X}$, if $n=p u+j(0<j<p)$ 
Note again that the definition of $\mathcal{M}$ is nondeterministic. This is not a problem, since we define that function by its semantics, and for all $p$ in $\gamma(n)$ the functions are semantically equivalent. On the other hand, since elements of Chains can be represented as words on our help functions, a real implementation chooses the minimal representation w.r.t. length.

For instance (for the dichotomic strategy)

$$
\begin{aligned}
\mathcal{M}_{87} & =\mathcal{C}_{87,10} \\
& =\mathcal{K}_{10,7} \circ \mathcal{M}_{8} \circ \mathbf{X} \\
& =\mathcal{K}_{7,3} \circ \mathbf{W}_{2} \circ \mathcal{M}_{1} \circ \mathbf{X} \circ \mathbf{Q} \circ \mathbf{Q} \circ \mathbf{Q} \circ \mathbf{X} \\
& =\mathcal{K}_{3,1} \circ \mathbf{W}_{2} \circ \mathcal{M}_{2} \circ \mathbf{X} \circ \mathbf{W}_{2} \circ \mathbf{X} \circ \mathbf{Q} \circ \mathbf{Q} \circ \mathbf{Q} \circ \mathbf{X} \\
& =\mathcal{M}_{1} \circ \mathbf{W} \circ \mathcal{M}_{3} \circ \mathbf{W}_{2} \circ \mathcal{M}_{2} \circ \mathbf{X} \circ \mathbf{W}_{2} \circ \mathbf{X} \circ \mathbf{Q} \circ \mathbf{Q} \circ \mathbf{Q} \circ \mathbf{X} \\
& =\mathbf{W} \circ \mathbf{W} \circ \mathbf{Q} \circ \mathbf{X} \circ \mathbf{W}_{2} \circ \mathbf{Q} \circ \mathbf{X} \circ \mathbf{W}_{2} \circ \mathbf{X} \circ \mathbf{Q} \circ \mathbf{Q} \circ \mathbf{Q} \circ \mathbf{X}
\end{aligned}
$$

The correctness of these definitions according to the definitions in 3.3 .1 can be proven easily by induction. The example of computation below shows the intuitive contents of the help functions $\mathbf{W}, \mathbf{W}_{\mathbf{2}}$, etc.

$$
\begin{aligned}
\mathcal{M}_{87} \mathbf{K}\langle x\rangle & =\left(\mathbf{W} \circ \mathbf{W} \circ \mathbf{Q} \circ \mathbf{X} \circ \mathbf{W}_{2} \circ \mathbf{Q} \circ \mathbf{X} \circ \mathbf{W}_{2} \circ \mathbf{X} \circ \mathbf{Q} \circ \mathbf{Q} \circ \mathbf{Q} \circ \mathbf{X}\right) \mathbf{K}\langle x\rangle \\
& =\left(\mathbf{W} \circ \mathbf{Q} \circ \mathbf{X} \circ \mathbf{W}_{\mathbf{2}} \circ \mathbf{Q} \circ \mathbf{X} \circ \mathbf{W} \circ \mathbf{X} \circ \mathbf{Q} \circ \mathbf{Q} \circ \mathbf{Q} \circ \mathbf{X}\right) \mathbf{K}\langle x, x\rangle \\
& =\left(\mathbf{Q} \circ \mathbf{X} \circ \mathbf{W} \mathbf{W}_{\mathbf{2}} \circ \mathbf{Q} \circ \mathbf{X} \circ \mathbf{W} \circ \mathbf{X} \circ \mathbf{Q} \circ \mathbf{Q} \circ \mathbf{Q} \circ \mathbf{X}\right) \mathbf{K}\langle x, x, x\rangle \\
& =\left(\mathbf{X} \circ \mathbf{W}_{\mathbf{2}} \circ \mathbf{Q} \circ \mathbf{X} \circ \mathbf{W}_{2} \circ \mathbf{X} \circ \mathbf{Q} \circ \mathbf{Q} \circ \mathbf{Q} \circ \mathbf{X}\right) \mathbf{K}\left\langle x^{2}, x, x\right\rangle \\
& =\left(\mathbf{W}_{\mathbf{2}} \circ \mathbf{Q} \circ \mathbf{X} \circ \mathbf{W}_{\mathbf{2}} \circ \mathbf{X} \circ \mathbf{Q} \circ \mathbf{Q} \circ \mathbf{Q} \circ \mathbf{X}\right) \mathbf{K}\left\langle x^{3}, x\right\rangle \\
& =\left(\mathbf{Q} \circ \mathbf{X} \circ \mathbf{W}_{\mathbf{2}} \circ \mathbf{X} \circ \mathbf{Q} \circ \mathbf{Q} \circ \mathbf{Q} \circ \mathbf{X}\right) \mathbf{K}\left\langle x^{3}, x, x^{3}\right\rangle \\
& =\left(\mathbf{X} \circ \mathbf{W}_{\mathbf{2}} \circ \mathbf{X} \circ \mathbf{Q} \circ \mathbf{Q} \circ \mathbf{Q} \circ \mathbf{X}\right) \mathbf{K}\left\langle x^{6}, x, x^{3}\right\rangle \\
& =(\mathbf{W} \circ \mathbf{X} \circ \mathbf{Q} \circ \mathbf{Q} \circ \mathbf{Q} \circ \mathbf{X}) \mathbf{K}\left\langle x^{7}, x^{3}\right\rangle \\
& =(\mathbf{X} \circ \mathbf{Q} \circ \mathbf{Q} \circ \mathbf{Q} \circ \mathbf{X}) \mathbf{K}\left\langle x^{7}, x^{3}, x^{7}\right\rangle \\
& =(\mathbf{Q} \circ \mathbf{Q} \circ \mathbf{Q} \circ \mathbf{X}) \mathbf{K}\left\langle x^{10}, x^{7}\right\rangle \\
& =(\mathbf{Q} \circ \mathbf{Q} \circ \mathbf{X}) \mathbf{K}\left\langle x^{20}, x^{7}\right\rangle \\
& =(\mathbf{Q} \circ \mathbf{X}) \mathbf{K}\left\langle x^{40}, x^{7}\right\rangle \\
& =\mathbf{X K}\left\langle x^{80}, x^{7}\right\rangle \\
& =\mathbf{K}\left\langle x^{87}\right\rangle \\
& =x^{87}
\end{aligned}
$$




\section{Notes on IMPLEMENTATION}

We have implemented our approach in the Scheme programming language $[11,17]$.

\subsection{Data representation}

The elements of Chains are represented as lists whose elements are $\mathbf{W}, \mathbf{W}_{\mathbf{2}}, \mathbf{Q}$ or $\mathrm{X}$. In the case of a deterministic strategy we have an efficient implementation on chains, by using destructive list operations.

\subsection{Chain optimization}

Chains, expressed in terms of $\mathbf{W}, \mathbf{W}_{\mathbf{2}}, \mathbf{X}$ and $\mathbf{Q}$ can be further optimized. For instance, the word associated with $\sigma_{1}$ begins with two occurrences of $\mathbf{W}_{2}$, corresponding to two saves on a stack of the same values. A register allocation pass translates the chain into a code of the same form as shown in figure 3.

\subsection{Encapsulation}

The optimized code is encapsulated in a functional Make-Expt, which takes as an argument an interpretation, i.e. a structure consisting of a domain $\mathbf{D}$ and procedures for multiplying and squaring elements of $D$, and returns a procedure which allows the computation of $n$th powers in $\mathrm{D}$.

It should be noted that, for a given $n$ and a given strategy, the generation of the same computation scheme for $x^{n}$ works for any number of interpretations.

\subsection{Practical results}

We have done experiments in the Chez-Scheme ${ }^{(C)}$ system. The first result shows the interest of our approach in the case where the multiplication is a rather complex operation.

We have compared in figure 6 the classical binary algorithm with our a pproach, in computing $(2 / 3)^{n}$ for all $1 \leq n \leq 4096$, using Chez-Scheme. For time and space, we take as unity the performances of the classical binary algorithm. The time statistics include all garbage collecting times. It must be noted that, on a Sun $3 / 80$, using Chez-Scheme, the time for generation of all chains (as functional objects) for $n$ varying from 2 up to 4096 was only $33 \mathrm{sec}$ (CPU time).

In the case of simple interpretations (like integers modulo $p$ ), both approaches give equivalent results, and sometimes the classical algorithm works faster. This can be explained by the fact that the entire program is written in Scheme, and the process of interpreting the code associated with a chain is too high-level.

We expect this time to decrease in a real implementation where the chain is compiled into machine code.

We also wrote a program which evaluates directly $x^{n}$ with a deterministic strategy. This program is a direct translation in Scheme of the equations in 3.3. Functions $\mathcal{M}, \mathcal{C}$ and $\mathcal{K}$ are uncurried, i.e. their definitions are of the form:

(lambda (n p kappa $x$ xstar) $\langle\ldots\rangle$ ). 
Since no intermediate object is generated, this last program runs faster than the one described above. This proves the adequacy of our equations, and illustrates the power of Continuation Passing Style.

\begin{tabular}{|c|c|c|c|}
\hline & CPU time & Real time & Allocation(bytes) \\
\hline Binary algorithm & 1.0000 & 1.0000 & 1.000 \\
Chain generation & 0.0033 & 0.0033 & 0.370 \\
Chain application & 0.8600 & 0.8700 & 1.084 \\
\hline
\end{tabular}

Fig. 6 Some statistics

\subsection{Chain widths}

The width of a chain determines the number of registers needed for the computation of $x^{n}$. Thus it is very important to know some properties of chain widths.

\subsubsection{Binary strategy}

The binary strategy generates chains of width less or equal than 2; this is easily proven by induction, and implies the well known iterative binary algorithm to compute $x^{n}$ by successive shifts, squares and products.

\subsubsection{Dichotomic Strategy}

This case is of major interest, since $\delta$ was found to be the most practical strategy for our approach.

Lemma 1. Let us denote by $\bar{m}(n), \bar{c}(n, p)$, and $\bar{k}(n, p)$, the respective widths of the computation schemes $M(x, n), C(x, n, p)$, and $K(x, n, p)$. The following inequalities are proved by a straightforward register analysis.

$$
\begin{aligned}
\bar{m}(3) & =2, \\
\bar{m}(n) & =1, \text { if } n=2^{a}, \\
\bar{m}(n) & =\bar{c}(n, \delta(n)), \text { otherwise; } \\
\bar{c}(n, p) & =\max (\bar{m}(p), \bar{m}(u)), \text { if } n=p u, \\
\bar{c}(n, p) & =\max (\bar{k}(p, j), 1+\bar{m}(u)), \text { if } n=p u+j ; \\
\max (\bar{m}(p), \bar{m}(u)) \leq \bar{k}(n, p) & \leq \max (\bar{m}(p), 1+\bar{m}(u)), \text { if } n=p u, \\
\max (\bar{k}(p, j), 2+\bar{m}(u)) \leq \bar{k}(n, p) & \leq \max (\bar{k}(p, j), 2+\bar{m}(u)), \text { if } n=p u+j .
\end{aligned}
$$

Theorem 1. The maximum width of a $\delta$-chain for $n$ is bounded by $\mathrm{O}\left(\log _{2}\left(\log _{2}(n)\right)\right)$. The maximum width of $\delta$-chains is unbounded.

Proof. The first assertion follows directly from lemma 1 : Since the strategy $\delta$ breaks its arguments into parts whose length (in binary representation) differ of at most 1 , one can iterate $\delta$ on $n$ only $\log _{2}\left(\log _{2}(n)\right)$ times. The evaluation of a bound for $\bar{m}(n)$ defines 
a max-tree, such that each branch has at most $\log _{2}\left(\log _{2}(n)\right)$ nodes labeled by $\bar{m}$. This implies that the number of " +1 " and " +2 " along a branch is bounded by that quantity.

We prove the second assertion by construction of an infinite sequence of $\delta$-chains of strictly increasing width. To do that, let us define the sequences $a_{i}(i \geq 0), b_{i}(i \geq 0)$, and $c_{i}(i \geq 0)$ by:

- $a_{0}=1 ; \quad b_{0}=2 ; \quad c_{0}=5$.

- $a_{i+1}=b_{i} ; \quad b_{i+1}=a_{i}+b_{i} \times c_{i} ; \quad c_{i+1}=b_{i}+b_{i+1} \times 2^{\lambda\left(b_{i+1}\right)}$

We prove by induction on $i$ the following inequalities:

- $a_{i}<b_{i}<c_{i}$. This follows directly from our definitions.

- $\delta\left(n_{i}\right)=b_{i}$. This results directly from the definition of the strategy $\delta$.

- $\bar{m}\left(c_{i}\right)=\bar{k}\left(b_{i}, a_{i}\right)$. This is true for $i=0$ (see figure 7). For $i>0$, this follows from the construction of $\mathcal{M}_{c_{i+1}}$ :

$$
\begin{aligned}
\mathcal{M}_{c_{i+1}}= & \mathcal{C}_{c_{i+1}, b_{i+1}} \\
= & \mathcal{K}_{b_{i+1}, b_{i}} \circ \mathcal{M}_{u_{i+1}} \circ \mathbf{X} \\
& \text { where } u_{i}=2^{\lambda\left(b_{i+1}\right)} .
\end{aligned}
$$
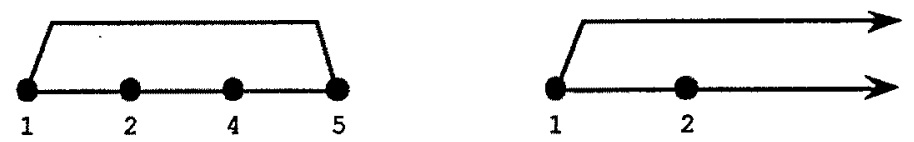

Fig. 7 Widths of $M_{5}$ and $K_{2,1}$

- $\bar{k}\left(b_{i+1}, a_{i+1}\right)>\bar{m}\left(c_{i}\right):$ This follows from the equalities:

$$
\begin{aligned}
\mathcal{K}_{b_{i+1}, a_{i+1}} & =\mathcal{K}_{b_{i+1}, b_{i}} \\
& =\mathcal{K}_{b_{i}, a_{i}} \circ \mathbf{W}_{\mathbf{2}} \circ \mathcal{M}_{c_{i}}
\end{aligned}
$$

Since $x^{a_{i}}<y$, we need at least one more register than $\bar{m}\left(c_{i}\right)$ (see figure 8).

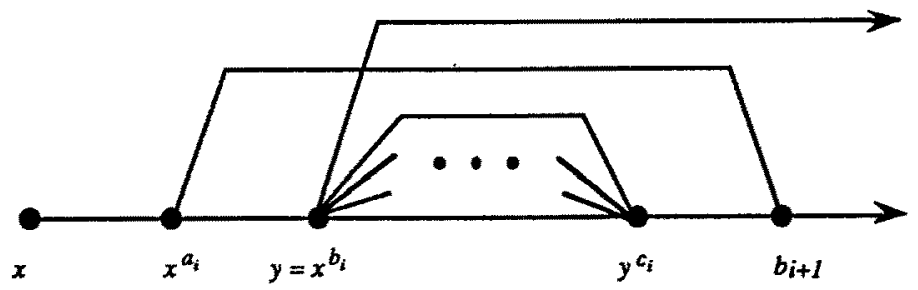

Fig. 8 Construction of $K\left(b_{i+1}, a_{i+1}\right)$ 
Figure 9 gives, for each width $w$ up to 6 , the first $n$ such that the chain for $n$ is exactly of width $n$.

\begin{tabular}{|r|r|}
\hline Width & $\mathrm{n}$ \\
\hline 1 & 2 \\
2 & 3 \\
3 & 11 \\
4 & 338 \\
5 & 1506 \\
6 & 4208642 \\
\hline
\end{tabular}

Fig. 9 First $\delta$-chains of given width

Figure 10 illustrates the construction in the proof of Theorem 1. Remark that for some $c_{i}, \bar{m}\left(c_{i}\right)>\log _{2}\left(\log _{2}\left(c_{i}\right)\right)$.

\begin{tabular}{|c|c|c|c|}
\hline$i$ & $c_{i}$ & $\bar{m}\left(c_{i}\right)$ & $\log _{2}\left(\log _{2}\left(c_{i}\right)\right)$ \\
\hline 1 & 5 & 2 & 2 \\
2 & 90 & 3 & 3 \\
3 & 507915 & 4 & 5 \\
4 & $\ldots$ & 6 & 6 \\
5 & $\ldots$ & 8 & 8 \\
6 & $\ldots$ & 10 & 10 \\
7 & $\ldots$ & 12 & 11 \\
8 & $\ldots$ & 14 & 13 \\
9 & $\ldots$ & 16 & 14 \\
\hline
\end{tabular}

Fig. 10 A strictly increasing sequence of $\delta$-chains

\subsection{Remarks on complexity}

Complexity of chain generation is discussed in [2]. The computation of all euclidean chains up to $n$, according to a given strategy $\gamma$, is achieved in time $\mathrm{O}\left(n \gamma_{n} \log ^{2} n\right)$, where $\gamma_{n}$ is the complexity of computing $\gamma(n)$. This takes into account the complexity of doing arithmetics with bignums (the factor $\log ^{2} n$ in the formula).

In the case of a deterministic strategy like $\delta, \gamma_{n}=\mathrm{O}(1)$. Therefore, the time of creation of all chains up to $n$ is $\mathrm{O}\left(n \log ^{2} n\right)$.

The analysis of the computation tree of a $\delta$-chain for a specific $n$ (as in the proof of Theorem 1) shows that the worst-case time for the creation of the corresponding $\delta$-chain is $\mathrm{O}\left(\log (\log (n)) \log ^{2} n\right)$. This formula clearly shows that computation schemes for $x^{n}$ can be generated very quickly (see section 4.4). Our approach is very well fitted to the case of a run-time compiler, as in many versions of Scheme. 


\section{CONCLUSION}

The merging of concepts from semi-numerical algorithmics and applicative programming semantics allowed us to give effective applications to the theory of addition chains.

Various extensions of this work are possible:

- Generalization to vectorial addition chains studied in [5].

- Study of strategies, in particular to give the possibility of changing the current strategy during the computation of a chain.

- Integration of our approach in a real Scheme Compiler. This would include the following features:

- The generated chains would be translated into machine language.

- An analyzer should decide wether to use addition chains or compute powers classically. (This choice depends on the complexity of products and squares, the size of the exponent, an estimation on the number of times the same computation scheme is reused, etc.) We imagine this can be done by a technique related to partial evaluation [4].

\section{References}

[1] F. Bergeron, J. Berstel, S. Brlek, A Unifying Approach to the Generation of Addition Chains, Proceedings of the XV Latino-American Conference on Informatics, 10-14 July (1989) 29-38.

[2] F. Bergeron, J. Berstel, S. Brlek, Efficient Computation of Addition Chains, Submitted.

[3] F. Bergeron, J. Berstel, S. Brlek, C. Duboc, Addition Chains using Continued Fractions, Journal of Algorithms (1988) 403-412.

[4] D. Bjørner, A.P. Ershov, N.D. Jones (eds.) Partial Evaluation and Mixed Computation, North Holland, (1988).

[5] F. Bergeron, J. Olivos, Vectorial Addition Chains Using Euclid's Algorithm, (1989) Submitted.

[6] A. Brauer, On Addition Chains, Bull.Amer.Math.Soc. 45, (1939) 736-739.

[7] S. Brlek, P. Castéran, R. Strandh, Chaînes d'additions et structures de contrôle, Journées JFLA 91, Gresse-en-Vercors, France, January 28-29, (1991).

[8] M.Castan, M.-H. Durand, M. Lemaitre, A Set of Combinators for Abstraction in Linear Space, Information Processing Letter 24, (1987) 183-188.

[9] H.B. Curry, R. Feys, Combinatory Logic, North Holland (1974).

[10] P. Downey, B.Leong, R.Sethi, Computing Sequences with Additions Chains, SIAM J.Computing 3, (1974) 1-10.

[11] R.K. Dybvig, The Scheme Programming Language, Prentice Hall (1987). 
[12] P. Fradet, D. Le Metayer, Compilation of Functional Languages by Program Transformation, INRIA Research, Report 1040 (1989).

[13] D.E. Knuth, The Art of Computer Programming, vol.2, Addison Wesley (1981).

[14] D. Kranz, R. Kelsey, J.A. Rees, P. Hudak, J. Philbin , N.I. Adams, Orbit: An Optimizing Compiler for Scheme, Proceedings of the SIGPLAN '86 Symposium on Compiler Construction, July 1986.

[15] A. Scholz, Jahresbericht, Deutsche Math.-Verein. 47, (1937) 41.

[16] J.E. Stoy, The Scott-Strachey Approach to Programming Language Theory, MIT Press, Cambridge (1977).

[17] G.L. Steele, G.J. Sussman, Scheme: an Interpreter for the Extended Lambdacalculus, Memo 349, MIT Artificial Intelligence Laboratory (1975).

[18] M. Wand, Continuation-Based Program Transformation Strategies, J. of Computer Languages, (1978) 241-263. 